\title{
Mitigating CFO in OFDM systems by exploring the symbol structure
}

\author{
Carlos Ribeiro ${ }^{\#, * 1}$, Atílio Gameiro ${ }^{\# 2}$ \\ \# Instituto de Telecomunicações / Universidade de Aveiro \\ Campo Universitário, 3810 - 193 Aveiro, Portugal \\ ${ }^{1}$ cribeiro@av.it.pt \\ 2 amg@ua.pt \\ *Instituto Politécnico de Leiria \\ Morro do Lena, Alto Vieiro, 2411 - 901 Leiria, Portugal
}

\begin{abstract}
In this paper we revisit the carrier frequency offset (CFO) estimation and removal for broadband orthogonal frequency division multiplexing (OFDM) systems. We propose an algorithm that explores the time-domain (TD) properties of transmitted symbols carrying pilots and data. A careful examination of the components that constitute the TD received symbols leads to the definition of a simple, yet effective, cost function. In a real scenario, the cost function presents a bowl shape with a single global minimum easily identifiable (no local minima). The proposed method is independent of the channel estimation and decision, presenting a very low computational load. The feasibility of the investigated method is substantiated by system simulation using BRAN-A broadband wireless channel model. Simulation results show that the impact of the residual CFO in the system's performance is minimal.
\end{abstract}

\section{INTRODUCTION}

Future mobile broadband applications will require reliable high data-rate wireless communication systems. In recent years, OFDM-based transmission systems [1]-[2] emerged as the scheme with the potential to fulfill these conditions, with bandwidth efficiency and robustness to frequency selective channels, common in mobile personal communication systems. Various forms of OFDM have been adopted in different standards: IEEE.802.11a/g [3], IEEE.802.16 [4], DAB/DVB [1]. However, the long symbol duration makes OFDM systems particularly sensitive to carrier frequency offsets (CFO) that always exist between the base station (BS) and mobile terminal (MT). The presence of CFO destroys the orthogonality among sub-carriers leading to inter-carrier interference (ICI), that causes severe degradation of the system's bit error rate (BER) [5]-[7].

The estimation and removal of the CFO has been the focus of a considerable number of works published in recent years. The algorithms can be categorized as blind or data-aided. The first category explores the properties of the received symbols (commonly the cyclic prefix (CP)) [8]-[10]. The data-aided algorithms use dedicated training symbols [11], [12] or exploit the presence of null sub-carriers [13], [14].

This paper contains a proposal for a CFO estimation and removal algorithm that exploits a standardized transmission format where constant-valued pilot symbols are regularly spread in every OFDM symbol. The time-domain (TD) properties of the symbols are examined and exploited to perform the CFO estimation. The CFO is compensated in TD, decoupled from the channel estimation and decision, leading to a low complexity scheme. No additional information is required to perform the task in hand (training symbols or null sub-carriers).

The paper is organized as follows. Next Section gives a brief introduction to the wireless multipath channel and the OFDM baseband model. In Section III, the proposed CFO estimation and removal algorithm is developed. The feasibility of the developed method is substantiated by simulation results presented in Section IV. Finally, conclusions are drawn in Section V.

\section{OFDM IN MOBILE WIRELESS CHANNELS}

Before introducing the investigated method, we will briefly overview the mobile wireless multipath channel and the considered OFDM baseband model.

Throughout the text, the notation $(\sim)$ is used for TD vectors and elements and its absence denotes frequencydomain (FD) vectors and elements. The index $n$ denotes TD elements and $k$ FD elements. Unless stated otherwise, the vectors involved in the transmission/reception process are column vectors with $N_{C}$ complex elements. The superscripts $(.)^{T}$ and $(.)^{H}$ denote transpose and Hermitian transpose, respectively.

\section{A. The Wireless Multipath Channel}

Let's consider that the system transmits over a multipath Rayleigh fading wireless channel, modeled by the discretetime channel impulse response (CIR)

$$
\tilde{h}[n]=\sum_{l=0}^{L_{p}-1} \alpha_{l} \delta\left[n-\tau_{l}\right]
$$

where $L_{p}$ is the number of channel paths, $\alpha_{l}$ and $\tau_{l}$ are the complex value and delay of path $l$, respectively. The paths are assumed to be statistically independent, with normalized average power, $\sum_{l=0}^{L_{p}-1} \sigma_{h}^{2}[l]=1$, where $\sigma_{h}^{2}[l]$ is the average power of path $l$. The channel is time-variant due to the motion 


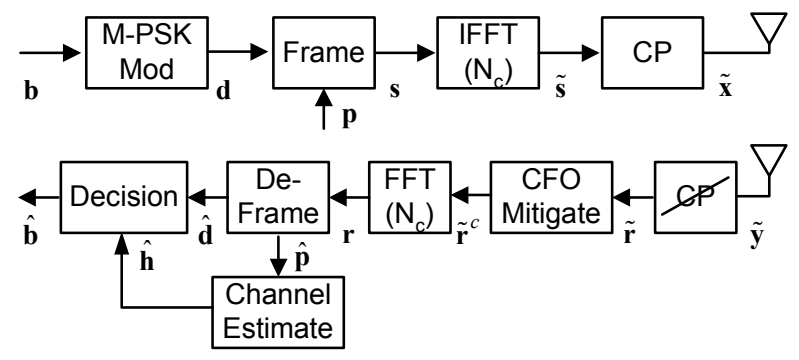

Fig. 1. OFDM baseband system model.

of the mobile terminal (MT), but we will assume that the CIR is constant during one OFDM symbol. The time dependence of the CIR is not present in the notation for simplicity. Assuming that the insertion of a long enough cyclic prefix (CP) in the transmitter assures that the orthogonality of the subcarriers is maintained after transmission, the channel frequency response (CFR) can be expressed as

$$
h[k]=\sum_{l=0}^{L_{p}-1} \alpha_{l} \exp \left[-j \frac{2 \pi}{N_{C}} k \tau_{l}\right],
$$

where $N_{C}$ is the total number of sub-carriers of the OFDM system.

\section{B. OFDM Baseband Model}

Let's consider the OFDM baseband system with $N_{C}$ subcarriers depicted in Fig. 1. At time $i$, the binary data vector $\mathbf{b}$ is $M$-ary PSK or QAM modulated into vector $\mathbf{d}$.

To assist in the channel estimation process, pilot symbols are multiplexed together with data symbols in different subcarriers. The pilot symbols are collected in vector $\mathbf{p}$.

Vectors $\mathbf{p}$ and $\mathbf{d}$ contain non-zero values at disjoint positions (sub-carriers). The resulting FD vector is $\mathbf{s}=\mathbf{d}+\mathbf{p}$.

The inverse discrete Fourier transform (DFT) block transforms the input vector into the TD vector $\tilde{\mathbf{s}}$, using an efficient $N_{C}$-points inverse fast Fourier transform (FFT) algorithm.

An $L$ samples long guard interval, in the form of $\mathrm{CP}$, is prefixed to vector $\tilde{\mathbf{s}}$, resulting in the TD transmitted vector

$$
\tilde{\mathbf{x}}=\mathbf{A}_{C P} \mathbf{F}^{H} \mathbf{s}=\mathbf{A}_{C P}(\tilde{\mathbf{d}}+\tilde{\mathbf{p}})
$$

where $\mathbf{F} \triangleq N_{C}^{-1 / 2}\left(e^{-j 2 \pi k n / N_{C}}\right)_{k, n=0,0}^{N_{C}-1, N_{C}-1}$ is the $N_{C} \times N_{C} \quad$ DFT matrix and $\mathbf{A}_{C P}=\left[\begin{array}{ll}\mathbf{I}_{N_{C}, L} & \mathbf{I}_{N_{C}}\end{array}\right]^{T}$ is the matrix that adds the CP, with $\mathbf{I}_{N_{C}}$ denoting the $N_{C} \times N_{C}$ identity matrix and $\mathbf{I}_{N_{C}, L}$ denoting the last $L$ columns of $\mathbf{I}_{N_{C}}$. The TD vectors $\tilde{\mathbf{d}}$ and $\tilde{\mathbf{p}}$ collect, respectively, the components of the data symbols and pilot symbols present in $\tilde{\mathbf{s}}$.

Let $w_{o}=2 \pi f_{o} \Delta t$ be the normalized angular CFO, where $f_{o}$ is the frequency offset due to the frequency mismatch of the oscillators of the transmitter and the receiver, and $\Delta t$ is the sampling interval.

The $n$-th received signal sample of the $i$-th symbol can be expressed as

$\tilde{y}_{i}[n]=\exp \left[j w_{o}\left(i\left(N_{C}+L\right)+n\right)\right] \sum_{l=0}^{L_{p}-1} \tilde{h}_{i}[l] \tilde{x}_{i}[n-l]+\overline{\tilde{n}}_{i}[n]$,

where $\overline{\tilde{n}}_{i}[n]$ is a sample of independent and identically distributed (iid) zero mean additive white Gaussian noise (AWGN) with variance $\sigma_{n}^{2}$. Collecting the $\left(N_{C}+L\right)$ samples of the symbol, it can be written as

$$
\tilde{\mathbf{y}}_{i}=\exp \left[j w_{o} i\left(N_{C}+L\right)\right] \mathbf{C}_{\left(N_{C}+L\right)}\left(w_{o}\right) \tilde{\mathbf{H}}_{i}^{l i n} \tilde{\mathbf{x}}_{i}+\overline{\tilde{\mathbf{n}}}_{i},
$$

where the vector $\overline{\tilde{\mathbf{n}}}_{i}$ collects the noise samples that affect the $i$-th symbol, the matrix $\tilde{\mathbf{H}}_{i}^{\text {lin }}$ is the $\left(N_{C}+L\right) \times\left(N_{C}+L\right)$ lower triangular Toeplitz channel convolution matrix with first column $\tilde{\mathbf{h}}_{i}$ (column $\left(N_{C}+L\right)$-vector with the discrete-time CIR (its elements are defined by (1)) padded with zeros) and the $\left(N_{C}+L\right) \times\left(N_{C}+L\right)$ diagonal matrix that holds the phase rotation that affects each symbol sample is

$\mathbf{C}_{\left(N_{C}+L\right)}\left(w_{o}\right)=\operatorname{diag}\left(\left[1 \exp \left(j w_{o}\right) \cdots \exp \left(j w_{o}\left(N_{C}+L-1\right)\right)\right]\right)(6)$

The receiver starts by removing the $\mathrm{CP}$ from the received symbol. The resulting vector is

$$
\begin{aligned}
\tilde{\mathbf{r}}_{i} & =\mathbf{R}_{C P} \tilde{\mathbf{y}}_{i}=\exp \left[j w_{o} i\left(N_{C}+L\right)\right] \mathbf{R}_{C P} \mathbf{C}_{\left(N_{C}+L\right)}\left(w_{o}\right) \tilde{\mathbf{H}}_{i}^{l i n} \tilde{\mathbf{x}}_{i}+\tilde{\mathbf{n}}_{i}, \\
& =\theta_{i} \mathbf{C}_{N_{C}}\left(w_{o}\right) \mathbf{R}_{C P} \tilde{\mathbf{H}}_{i}^{l i n} \mathbf{A}_{C P} \tilde{\mathbf{s}}_{i}+\tilde{\mathbf{n}}_{i}
\end{aligned}
$$

where $\mathbf{R}_{C P}=\left[\begin{array}{ll}\mathbf{0}_{\left(N_{C} \times L\right)} & \mathbf{I}_{N_{C}}\end{array}\right]$ is the matrix that removes the CP with $\mathbf{0}_{\left(N_{C} \times L\right)}$ representing a $\left(N_{C} \times L\right)$ null matrix, $\tilde{\mathbf{n}}_{i}=\mathbf{R}_{C P} \overline{\tilde{\mathbf{n}}}_{i}$ is the resulting $\mathrm{TD}$ noise column $N_{C}$-vector, $\theta_{i}=\exp \left[j w_{o}\left(i\left(N_{C}+L\right)+L\right)\right]$ is the common phase that affects all samples of the $i$-th symbol and

$$
\mathbf{C}_{N_{C}}\left(w_{o}\right)=\operatorname{diag}\left(\left[1 \exp \left(j w_{o}\right) \cdots \exp \left(j w_{o}\left(N_{C}-1\right)\right)\right]\right)
$$

The last step in (7) was possible considering the structure of the matrices involved,

$$
\mathbf{R}_{C P} \mathbf{C}_{\left(N_{C}+L\right)}\left(w_{o}\right)=\exp \left[j w_{o} L\right] \mathbf{C}_{N_{C}}\left(w_{o}\right) \mathbf{R}_{C P} .
$$

With the assumption that there is no inter-symbol interference (ISI), $L \geq L_{p}$, equation (7) can be written as

$$
\begin{aligned}
\tilde{\mathbf{r}}_{i} & =\theta_{i} \mathbf{C}_{N_{C}}\left(w_{o}\right) \tilde{\mathbf{H}}_{i}^{c i r c} \tilde{\mathbf{s}}_{i}+\tilde{\mathbf{n}}_{i} \\
& =\theta_{i} \mathbf{C}_{N_{C}}\left(w_{o}\right) \mathbf{F}^{H} \mathbf{H}_{i} \mathbf{s}_{i}+\tilde{\mathbf{n}}_{i}{ }^{\prime}
\end{aligned}
$$


where $\tilde{\mathbf{H}}_{i}^{\text {circ }}=\mathbf{R}_{C P} \tilde{\mathbf{H}}_{i}^{\text {lin }} \mathbf{A}_{C P}$ is the $N_{C} \times N_{C}$ circulant matrix with circulant vector $\tilde{\mathbf{h}}_{i}$ and, due to the properties of the DFT, $\mathbf{H}_{i}=\mathbf{F} \tilde{\mathbf{H}}_{i}^{\text {circ }} \mathbf{F}^{H}=\operatorname{diag}\left(\mathbf{h}_{i}\right)$ with the elements of $\mathbf{h}_{i}$ defined by (2).

The CFO mitigate block estimates the CFO that affects the signal samples and mitigates it. Its operation can be described by

$$
\tilde{\mathbf{r}}_{i}^{c}=\theta_{i} \mathbf{C}_{N_{C}}^{H}\left(\hat{w}_{o}\right) \mathbf{C}_{N_{C}}\left(w_{o}\right) \mathbf{F}^{H} \mathbf{H}_{i} \mathbf{s}_{i}+\mathbf{C}_{N_{C}}^{H}\left(\hat{w}_{o}\right) \tilde{\mathbf{n}}_{i},
$$

where $\hat{w}_{o}$ is the estimated CFO. It is clear that if $\hat{w}_{o}=w_{o}$, $\mathbf{C}_{N_{C}}^{H}\left(\hat{w}_{o}\right) \mathbf{C}_{N_{C}}\left(w_{o}\right)=\mathbf{I}_{N_{C}}$ and the CFO is completely removed.

The DFT block transforms vector $\tilde{\mathbf{r}}^{c}$ to FD with an efficient FFT operation. Assuming that the CFO is completely eliminated, the resulting FD column $N_{C}$-vector can be expressed as

$$
\mathbf{r}_{i}=\mathbf{F} \tilde{\mathbf{r}}_{i}^{c}=\theta_{i} \mathbf{H}_{i} \mathbf{s}_{i}+\mathbf{n}_{i},
$$

where $\mathbf{n}_{i}=\mathbf{F} \mathbf{C}_{N_{C}}^{H}\left(\hat{w}_{o}\right) \tilde{\mathbf{n}}_{i}$ is the resulting FD noise vector.

The de-framing block separates the signals in the subcarriers conveying pilots and data symbols. The values in the pilot sub-carriers are collected in vector $\hat{\mathbf{p}}$ and fed to the channel estimation block. This block generates the CFR estimate for all sub-carriers, $\hat{\mathbf{h}}_{i}$. The phase-rotation $\theta_{i}$ is naturally removed in the estimation process, assuming that the pilot-aided scheme calculates the least squares (LS) estimates (back-rotated received signal).

The values in the data sub-carriers are collected in vector $\hat{\mathbf{d}}$ and fed to the decision block. Together with the channel estimate, the decision block is now able to decide what were the transmitted symbols, according to some decision rule, and generate the estimate of the transmitted data $\hat{\mathbf{b}}$.

\section{CFO ESTIMATION BY EXPLORING THE TD SYMBOL STRUCTURE}

Let's consider the common symbol structure where constant-valued pilots are regularly spread in the symbol every $N_{f}$ sub-carriers (i.e., the set of sub-carriers $\wp=\left\{0, N_{f}, 2 N_{f}, \cdots, N_{C}-N_{f}\right\}$ is dedicated to convey pilot symbols) and let's impose the additional condition on the pilot distance, $\frac{N_{C}}{N_{f}}=N_{t} \in \mathbb{N}$.

The output of the CFO mitigate block, $\tilde{\mathbf{r}}^{c}$, contains components from both the data samples in $\tilde{\mathbf{d}}$ and the pilot samples in $\tilde{\mathbf{p}}$. Due to the symbol structure and DFT properties, the pilot-dependent samples in $\tilde{\mathbf{r}}^{c}$ can be expressed by

$$
\tilde{r}^{c, p}[n]=\frac{\theta \exp \left[j\left(w_{o}-\hat{w}_{o}\right) n\right]}{N_{f}} \sum_{m=0}^{N_{f}-1} \tilde{h}\left[n-m N_{t}\right] .
$$



Fig. 2. Received pilot component $\tilde{\mathbf{r}}^{p}\left(N_{f}=8 ; N_{t}=128\right)$.

Equation (13) shows clearly that the pilot-dependent samples are limited to $N_{f}$ sets of $L_{p}$ samples, evenly spaced in the symbol every $N_{t}$ samples ( $N_{f}$ phase-shifted scaled replicas of the CIR). If the pilot separation fulfils the sampling theorem, the CIR replicas will not overlap, leaving some samples in-between replicas with no pilot-dependent energy. An example is depicted in Fig. 2. On the other hand, the datadependent component spans throughout the entire symbol.

Consider the $N_{t} \times N_{C}$ matrix $\mathbf{T}=\left[\begin{array}{lll}\mathbf{I}_{N_{t}} & \cdots & \mathbf{I}_{N_{t}}\end{array}\right]$ that operates on the candidate output of the CFO mitigate block. This operator sums the samples in the same position in all $N_{f}$ segments of $N_{t}$ samples, $\mathbf{j}=\mathbf{T} \tilde{\mathbf{r}}^{c}$. After this operation, the pilot-dependent component is limited to the set $\left\{L_{p}\right\}$ (with $L_{p}$ elements) where the CIR has energy. The remaining samples will only depend on the transmitted data and noise. Let's define the column $\left(N_{t}-L_{p}\right)$-vector that collects these samples, $\bar{j}[n]=j[n]$,if $n \notin\left\{L_{p}\right\}$. The $n$-th element of the vector $\overline{\mathbf{j}}$ for $i$-th received symbol is

$$
\begin{aligned}
& \bar{j}_{i}[n]=\sum_{m=0}^{N_{f}-1} \tilde{r}_{i}^{c}\left[n+m N_{t}\right]=\theta_{i} \sum_{m=0}^{N_{f}-1} e^{j\left(w_{o}-\hat{w}_{o}\right)\left(n+m N_{t}\right)} \\
& \times \sum_{l=0}^{L_{p}-1} \tilde{h}_{i}[l] \tilde{d}_{i}\left[n+m N_{t}-l\right]+\tilde{v}_{i}[n] \\
& =N_{C}^{-1 / 2} \theta_{i} e^{j\left(w_{o}-\hat{w}_{o}\right) n} \sum_{m=0}^{N_{f}-1} e^{j\left(w_{o}-\hat{w}_{o}\right) m N_{t}} \sum_{l=0}^{L_{p}-1} \tilde{h}_{i}[l] \\
& \times \sum_{\substack{k=0 \\
k \notin \mathfrak{Q}}}^{N_{C}-1} d_{i}[k] \exp \left[j 2 \pi k \frac{n+m N_{t}-l}{N_{C}}\right]+\tilde{v}_{i}[n] \\
& =N_{C}^{-1 / 2} \theta_{i} e^{j\left(w_{o}-\hat{w}_{o}\right) n} \sum_{l=0}^{L_{p}-1} \tilde{h}_{i}[l] \sum_{\substack{k=0 \\
k \notin \mathcal{B}}}^{N_{C}-1} d_{i}[k] \exp \left[j 2 \pi k \frac{n-l}{N_{C}}\right] \\
& \times \sum_{m=0}^{N_{f}-1} \exp \left[\frac{j 2 \pi m}{N_{f}}\left(N_{C}\left(f_{o}-\hat{f}_{o}\right) \Delta t+k\right)\right]+\tilde{v}_{i}[n]
\end{aligned}
$$

where $\hat{f}_{o}$ is the candidate frequency offset and the resulting noise component after the operation is

$$
\tilde{v}_{i}[n]=\sum_{m=0}^{N_{f}-1} \exp \left[-j \hat{w}_{o}\left(n+m N_{t}\right)\right] \tilde{n}_{i}\left[n+m N_{t}\right] .
$$

A careful inspection of (14) reveals that the data-dependent component is zero for 


$$
\left\{\phi=N_{C}\left(f_{o}-\hat{f}_{o}\right) \Delta t+k: \phi \in \mathbb{Z} \wedge \phi \notin \wp\right\},
$$

independently of the considered element of $\overline{\mathbf{j}}$. Keeping in mind that $k \notin \wp$, the solution for (16) is

$$
N_{C}\left(f_{o}-\hat{f}_{o}\right) \Delta t=m N_{f} \Leftrightarrow\left(f_{o}-\hat{f}_{o}\right)=\frac{m N_{f}}{N_{C} \Delta t}, m \in \mathbb{Z} .
$$

Equation (17) shows the condition for all the elements of $\overline{\mathbf{j}}$ to be simultaneously reduced to a noise component with the same variance $N_{f} \sigma_{n}^{2}$. It should be noted that it includes the desired condition when the CFO is completely eliminated, $\left(f_{o}-\hat{f}_{o}=0\right)$. A similar analysis reveals that the datadependent component has maximum magnitude for

$$
\begin{aligned}
& \left\{\gamma=N_{C}\left(f_{o}-\hat{f}_{o}\right) \Delta t: \gamma \in \mathbb{Z} \wedge \gamma \notin \wp\right\} \\
& \Rightarrow\left(f_{o}-\hat{f}_{o}\right)=\frac{m}{N_{C} \Delta t}, m \in \mathbb{Z} \wedge m \notin \wp
\end{aligned}
$$

With the definition and properties of $\overline{\mathbf{j}}$, defining the cost function $J(w)$ as the energy in $\overline{\mathbf{j}}$,

$$
J(w)=\overline{\mathbf{j}}^{H} \overline{\mathbf{j}},
$$

where $w$ represents the candidate $\mathrm{CFO}$, will result in a bowlshaped cost function with a single minimum if $\left(w_{o}-w\right)$ keeps with-in the interval limited by the maximum values (defined by (18)) that surround the minimum value, corresponding to no CFO.

The noise component in $\overline{\mathbf{j}}$ will impose a floor in the cost function that rapidly tends to $b N_{f} \sigma_{n}^{2}$ (and will be independent of the remaining $\mathrm{CFO},\left(w_{o}-w\right)$ ), as the number of samples, $b$, used in the estimation process increases.

The selection of the samples that make-up $\overline{\mathbf{j}}$ can be performed using an estimation procedure, but the simpler solution of using $\bar{j}[n]=j[n]$, if $n \in\left\{L, \cdots, N_{t}\right\}$ proved quite adequate, not compromising the system's performance and was adopted in the simulations.

The CFO estimate can be found using a simple gradient search that finds the minimum of $J(w)$,

$$
\hat{w}_{o}=\arg \left\{\min _{w} J(w)\right\} .
$$

As indicated by (17), the solution to (20) is not unique, but in a real system the CFO should remain inside the desired interval (around $\left.\left(w_{o}-w\right)=0\right)$ ). Fig. 3 depicts an example of the cost function for a system with $N_{C}=1024$ sub-carriers, sampling interval $\Delta t=10 \mathrm{~ns}$ and pilot separation $N_{f}=4$. The values in the plot were acquired using an $S N R=20 d B$. In Fig.

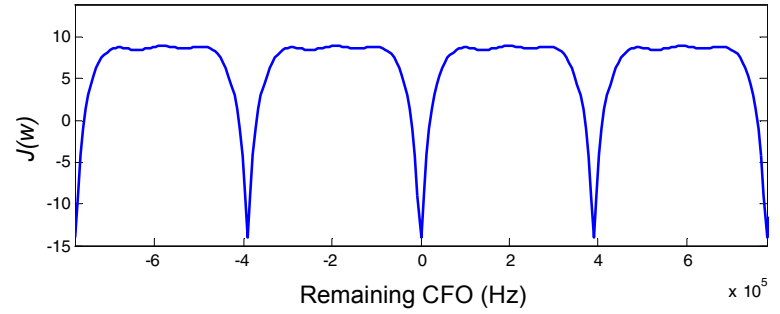

a)

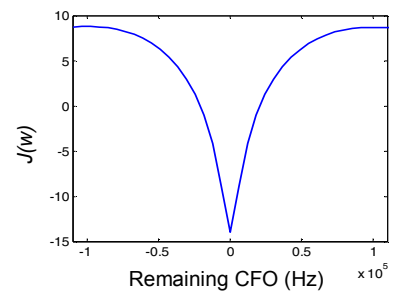

b)

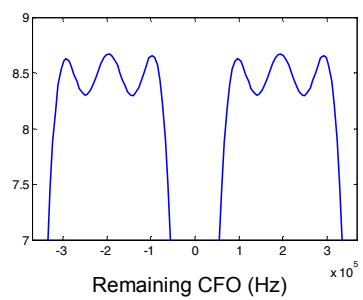

c)
Fig. 3. The cost function $J(w)$.

3.a) is visible the separation of $\approx 390 \mathrm{kHz}$ between consecutives minimum values. Fig. 3.b) shows in detail the interval around $\left(w_{o}-w\right)=0$. It is clear that it has a unique global minimum that is easy to find (no problem with local minimum values). In Fig. 3.c), the $N_{f}-1$ maximum values between consecutive minimum values are clearly visible. It also shows in detail that the separation of the maximum values around $\left(w_{o}-w\right)=0$ is $\approx 195 \mathrm{kHz}$.

\section{SIMULATION RESULTS}

A simulation scenario was implemented where $N_{C}=1024$ sub-carriers were QPSK modulated. The system's sampling interval was set to $\Delta t=10 \mathrm{~ns}$. The transmitted OFDM symbols carried pilots and data multiplexed according with the conditions previously defined, with a pilot separation $N_{f}=4$. The OFDM frame consists of 24 symbols. The symbols were transmitted over BRAN-A channels [15].

The $\mathrm{CFO}$ value was randomly generated in each frame with a value inside the interval $\left[-\left(N_{C} \Delta t\right)^{-1},\left(N_{C} \Delta t\right)^{-1}\right]$. The CFO estimation and removal was performed on a frame basis.

Fig. 4 shows the remaining CFO at the output of the CFO mitigate block. The dashed line represents the average remaining CFO over just 10 frames. The bar on each value of $\mathrm{Eb} / \mathrm{N} 0$ represents the standard deviation of the CFO estimate. The search for the cost function's minimum value was stopped when the gradient search reached a step of $100 \mathrm{~Hz}$, to keep a low computational load. The results show that the method is unbiased for the all range of Eb/N0 values and the algorithm generates estimates with minimal deviation from the true value.

Fig. 5 shows the system's BER when using the channel estimation method in [16]. It is evident that the impact of the residual CFO is minimal in the range of simulated values. 


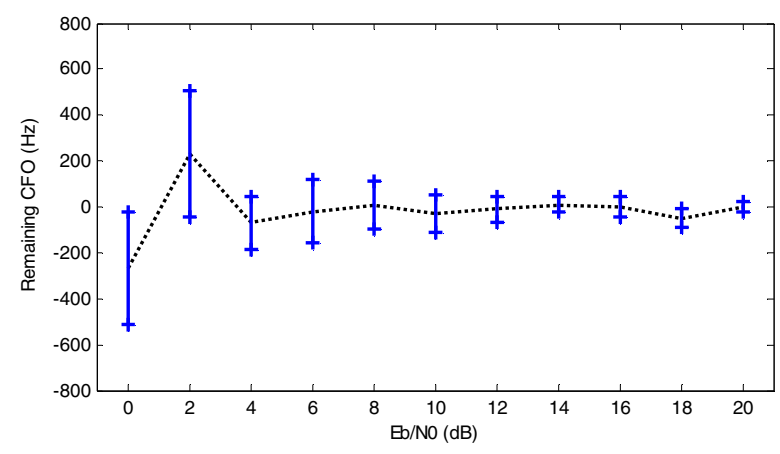

Fig. 4. Remaining CFO vs. Eb/NO.

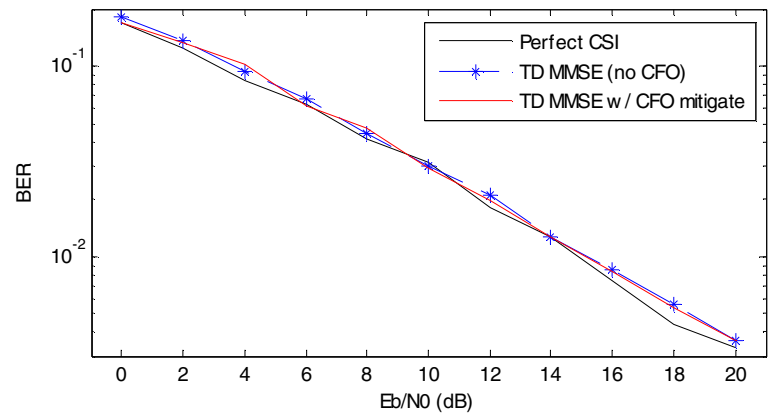

Fig. 5. System BER performance.

\section{CONCLUSIONS}

We have presented a CFO estimation and removal algorithm for OFDM that explores the TD structure of transmitted symbols carrying pilots and data. The algorithm relies solely on the data component present on the symbols to estimate the CFO, avoiding additional overhead like training symbols or null sub-carriers. The cost function presents multiple minimums but in a real scenario (with reasonably limited CFO span) it may be considered as a bowl-shaped function with a single global minimum easily identifiable. Thus, the algorithm can be used to both acquisition and tracking of CFO. Simulation results show that the residual CFO has a minimal impact in the system's performance, confirming that the CFO estimates have minimal deviation from the true value. The definition and shape of the cost function determine a very low complexity scheme. In addition, the proposed method is independent of the channel estimation and decision, resulting in a simple receiver structure.

\section{ACKNOWLEDGMENT}

The authors wish to thank Fundação para a Ciência e a Tecnologia and the European Union $7^{\text {th }}$ Research Framework Programme that partially supported this work through the projects "PHOTON - Distributed and Extendible Heterogeneous Radio Architectures using Fibre Optic Networks" (PTDC/EEA-TEL/72890/2006) and "CODIV Enhanced Wireless Communication Systems Employing COoperative DIVersity" (FP7-ICT-215477).

\section{REFERENCES}

[1] R. V. Nee, R. Prasad, OFDM for Wireless Multimedia Communications, 1st edition, Artech House, 2000.

[2] G. L. Stuber, J. R. Barry, S. W. McLaughlin, Y. Li, M. A. Ingram and T. G. Pratt, "Broadband MIMO-OFDM wireless communications," Proceedings of the IEEE, vol. 92, no. 2, pp. 271-294, Feb. 2004.

[3] Wireless LAN Medium Access Control (MAC) and Physical Layer (PHY) Specifications: High-Speed Physical Layer in the $5 \mathrm{GHz}$ Band, IEEE Std 802.11, 1999.

[4] I. Koffman, V. Roman, "Broadband wireless access solutions based on OFDM access in IEEE 802.16," IEEE Communications Magazine, vol. 40, no. 4, pp. 96-103, Apr. 2002.

[5] K. Sathananthan, C. Tellambura, "Probability of error calculation of OFDM systems with frequency offset," IEEE Transactions on Communications, vol. 49, no. 11, pp. 1884-1888, 2001.

[6] T. Pollet, M. Bladel, M. Moeneclaey, "BER sensitivity of OFDM systems to carrier frequency offset and Wiener phase noise," IEEE Transactions on Communications, vol. 43, pp. 191-193, 1995.

[7] L. Rugini, P. Banelli, "BER of OFDM systems impaired by carrier frequency offset in multipath fading channels," IEEE Transactions on Wireless Communications, vol. 4, no. 5, pp. 2279-2288, 2005.

[8] J.-J. Beek, M. Sandell, P. O. Börjesson, "ML estimation of time and frequency offset in OFDM systems," IEEE Transactions on Signal Processing, vol. 45, pp. 1800-1805, July 1997.

[9] H. Bolcskei, "Blind high-resolution uplink synchronization of OFDMbased multiple access schemes," in Proc. IEEE Workshop Signal Processing Advances in Wireless Communications, pp. 166-169, USA, 1999.

[10] N. Lashkarian, S. Kiaei, "Class of cyclic-based estimators for frequency-offset estimation of OFDM systems," IEEE Transactions on Communications, vol. 48, pp. 2139-2149, Dec. 2000.

[11] T. M. Schmidl, D. C. Cox, "Robust frequency and timing synchronization for OFDM," IEEE Transactions on Communications, vol. 45 , no. 12 , pp. 1613-1621, Dec. 1997.

[12] M. Morelli, U. Mengali, "An improved frequency offset estimator for OFDM applications," IEEE Communication Letters, vol. 3, pp. 75-77, March 1999.

[13] H. Liu, U. Tureli, "A high efficiency carrier estimator for OFDM communications," IEEE Communications Letters, vol. 2, pp. 104-106, April 1998.

[14] X. Ma, C. Tepedelenlioglu, G. B. Giannakis, S. Barbarossa, "Nondata-aided carrier offset estimations for OFDM with null subcarriers: Identifiability, algorithms, and performance," IEEE Journal on Selected Areas in Communications, vol. 19, pp. 2504-2515, Dec. 2001.

[15] Broadband Radio Access Networks (BRAN): HIPERLAN Type 2: Technical specification; Physical layer, ETSI TS 101475 V1.2.2, 2001.

[16] J.-J. van de Beek, O. Edfors, M. Sandell, S. K. Wilson, P. O. Borjesson, "On channel estimation in OFDM systems," in Proc. IEEE Vehicular Technology Conference, pp. 815 - 819, USA, July 1995. 